\title{
STABILITY AND ERROR OF THE VARIABLE TWO-STEP BDF FOR SEMILINEAR PARABOLIC PROBLEMS
}

\author{
ETIENNE EMMRICH
}

\begin{abstract}
The temporal discretisation of a moderate semilinear parabolic problem in an abstract setting by the two-step backward differentiation formula with variable step sizes is analysed. Stability as well as optimal smooth data error estimates are derived if the ratios of adjacent step sizes are bounded from above by 1.91 .
\end{abstract}

AMS Mathematics Subject Classification : 65M12, 65J15, 35K90, 47J35 Key words and phrases : Semilinear parabolic problem, time discretisation, backward differentiation formula, non-uniform grid, stability, error estimate

\section{Introduction}

Whereas multistep methods with variable step sizes are widely used in numerical computations, their analysis is still not complete. Because of the non-uniform grid, non-constant coefficients appear in the resulting scheme. Theoretical tools developed for difference equations with constant coefficients are therefore not applicable. Among the abundance of methods, the backward differentiation formulae (BDF) seem to be of particular interest. Especially the two-step BDF, which is strongly A-stable for constant time steps and of second order, plays an important rôle in the integration of non-stationary problems.

Stability and convergence of the variable two-step BDF has been studied by Grigorieff in a series of papers [8, 9, 10]. In particular, zero-stability has been shown for step size ratios less than $1+\sqrt{2} \approx 2.414$. For ratios bounded from above by $(1+\sqrt{3}) / 2 \approx 1.366, \mathrm{~A}_{0}$-stability as well as optimal error estimates in the case of a linear parabolic problem in a Hilbert space setting with a selfadjoint, strongly positive operator have been proven. Becker [1] could improve

Received September 28, 2004.

(C) 2005 Korean Society for Computational \& Applied Mathematics and Korean SIGCAM. 
the bound up to $(2+\sqrt{13}) / 3 \approx 1.868$. However, the stability and error constant may then depend on the sequence of step size ratios. The problem under consideration in [1] is again a linear parabolic one with a second order elliptic differential operator that might be time-dependent. $\mathrm{A}(\theta)$-stability type results with $\theta \leq \pi / 3$ have been provided in Grigorieff [9]. From these results, smooth as well as non-smooth data error estimates of optimal order could be derived for a linear homogeneous evolution equation in a Banach space setting with an operator that is the infinitesimal generator of a holomorphic semigroup. A smooth data error estimate in a similar situation, but with quite more restrictive assumptions on the partition of the time interval, has been obtained in Le Roux [11]. Stability estimates for rational multistep methods on uniform and quasi-uniform time grids approximating again a holomorphic semigroup can be also found in Palencia/García-Archilla [17]. So far, the time discretisation of nonlinear problems by means of the two-step BDF with variable time steps has not been considered, except the zero-stability result by Grigorieff [8] that also applies to Lipschitz-continuous nonlinear perturbations.

Other time discretisation schemes, single- as well as multistep methods with constant and sometimes with variable time steps, have been studied for linear problems by many authors. For an overview, we refer to Thomée [20], Fujita/Suzuki [6], and the references cited therein. Single-step methods applied to semilinear problems have been considered for instance in Crouzeix/Thomée [2], Slodička [18, 19], Lubich/Ostermann [13, 14], Ostermann/Thalhammer [16], and González et al. [7]. In Estep/Larsson [5], the discontinuous Galerkin method has been used for the time discretisation of semilinear problems. The two-step BDF with constant step sizes applied to a nonlinear problem has been considered in Zlámal [22]. Recently, we have analysed its application to the incompressible Navier-Stokes problem (cf. [3, 4]).

In this paper, we are concerned with the time discretisation of the abstract semilinear parabolic problem

$$
u^{\prime}+A u+g(u)=f, \quad u(0)=u_{0},
$$

where $A: V \rightarrow V^{*}$ is defined via $\langle A u, v\rangle=a(u, v)$ by a continuous, strongly positive bilinear form $a(\cdot, \cdot): V \times V \rightarrow \mathbb{R}$ on a real Hilbert space $V$ with its dual $V^{*}$, and $g: V \rightarrow V^{*}$ is some moderate nonlinearity specified below. Relying on Hilbert space methods, we shall derive stability and optimal smooth data error estimates for the second order variable two-step BDF in natural norms. For this, let the time interval $[0, T]$ for given $N \in \mathbb{N}$ be partitioned via

$$
0=t_{0}<t_{1}<\cdots<t_{N}=T, \tau_{n}:=t_{n}-t_{n-1}, r_{n}:=\tau_{n} / \tau_{n-1} .
$$

Throughout this paper, we assume that $r_{n}<R$ with $1<R<1+\sqrt{2}$. For suitably given values $u^{0}, u^{1}$ and approximations $f^{n} \sim f\left(t_{n}\right)$, we consider the nonlinear time-discrete problem

$$
\mathrm{D}_{2} u^{n}+A u^{n}+g\left(u^{n}\right)=f^{n}, \quad n=2, \ldots, N,
$$


to compute approximations $u^{n} \sim u\left(t_{n}\right)$ with the backward divided difference

$$
\mathrm{D}_{2} u^{n}:=\left(1+\frac{r_{n}}{1+r_{n}}\right) \mathrm{D}_{1} u^{n}-\frac{r_{n}}{1+r_{n}} \mathrm{D}_{1} u^{n-1}, \quad \mathrm{D}_{1} u^{n}:=\frac{u^{n}-u^{n-1}}{\tau_{n}} .
$$

Note that

$$
\mathrm{D}_{2} u^{n}=\frac{1}{\tau_{n}}\left(\frac{1+2 r_{n}}{1+r_{n}} u^{n}-\left(1+r_{n}\right) u^{n-1}+\frac{r_{n}^{2}}{1+r_{n}} u^{n-2}\right),
$$

and for an equidistant partition with $\tau_{n} \equiv \tau$, we have the well-known formula

$$
\mathrm{D}_{2} u^{n}=\frac{1}{\tau}\left(\frac{3}{2} u^{n}-2 u^{n-1}+\frac{1}{2} u^{n-2}\right) .
$$

If $r_{n}=0$ then, formally, $\mathrm{D}_{2}$ degenerates to $\mathrm{D}_{1}$, which corresponds to an occasional Euler-step in the computation.

Our stability and error estimates can be obtained for step size ratios less than $\bar{R} \approx 1.910$, where $\bar{R}$ is a root of

$$
\psi(R):=(R+1)^{4}-9 R(R-1)^{2}\left(R+\frac{1}{3}\right)^{2} .
$$

This also improves slightly Becker's bound. As in Becker [1], the appearing stability and error constant may depend, besides the usual exponential dependence on the time $T$ and problem parameters, on the sequence of step size ratios through $\exp \left(c \Gamma_{N}\right)$ with some $c>0$ independent on problem parameters and

$$
\Gamma_{N}:=\sum_{j=2}^{N-2}\left[r_{j+2}-r_{j}\right]_{-}, \quad[a]_{-}:=(|a|-a) / 2 .
$$

Note that $\Gamma_{N}=0$ if $\left\{r_{n}\right\}$ is monotonically increasing. Regarding the upper bound on the ratio of adjacent step sizes, we remark that the method of proof relies, more or less, upon considering each time step separately. We believe that the restriction on the step size ratios is only sufficient. A necessary condition for stability and error estimates to hold should rely upon a suitable combination of all ratios rather than on each ratio separately, as is observed in Grigorieff [8] with respect to the zero-stability.

As usual, we have to require some regularity of the exact solution for proving smooth data error estimates. In any case, such regularity leads to higher compatibility conditions on the data (cf. Temam [15]). Even for linear problems, such conditions can be hard to fulfil depending on the concrete space $V$, as is for instance the case for the Stokes problem because of the incompressibility condition that is contained in $V$. However, the only non-smooth data error estimate for a general grid we know of is due to Grigorieff [9] in the linear homogeneous case. Becker [1] has also derived non-smooth data error estimates for a special grid. It remains open to find a path to take advantage of the parabolic smoothing property in the non-homogeneous and semilinear case.

We do not consider a fully discrete problem as the results are independent of a possible spatial approximation. 
The paper is organised as follows: In Section 2, we give a precise description of the problem and its discretisation. In Section 3, we derive stability and error estimates for the linear case. The nonlinear case is studied in Section 4. A discrete Gronwall lemma is provided in Appendix A. Solvability of the original and time-discrete problem is proved in Apendix B.

\section{The semilinear problem and its time discretisation}

Let $V \subseteq H \subseteq V^{*}$ be a Gelfand triple, where $(V,\|\cdot\|)$ is a real Hilbert space that is dense and continuously embedded in the Hilbert space $(H,(\cdot, \cdot),|\cdot|)$. The dual $V^{*}$ of $V$ is equipped with the usual norm $\|f\|_{*}:=\sup _{v \in V \backslash\{0\}}\langle f, v\rangle /\|v\|$, where $\langle\cdot, \cdot\rangle$ denotes the dual pairing. In particular, there is some $\alpha>0$ such that

$$
|v| \leq \alpha\|v\| \quad \forall v \in V, \quad\|f\|_{*} \leq \alpha|f| \quad \forall f \in H .
$$

Due to the properties of $a(\cdot, \cdot)$, there are constants $\beta \geq \mu>0$ with

$$
|a(u, v)| \leq \beta\|u\|\|v\|, \quad a(v, v) \geq \mu\|v\|^{2}
$$

for all $u, v \in V$. For the skew-symmetric part of $a(\cdot, \cdot)$, we assume

$$
|a(u, v)-a(v, u)| \leq \gamma\|u\||v|
$$

for all $u, v \in V$ with some $\gamma \geq 0$. If $a(\cdot, \cdot)$ is symmetric then $\gamma=0$. The foregoing properties of the underlying spaces and bilinear form will be assumed tacitly.

Let $\mathcal{B}_{M}:=\{v \in V:|v| \leq M\}$ for given $M>0$ and let $g: V \rightarrow V^{*}$ be a (possibly nonlinear) function that satisfies the following structural assumptions we will recall explicitly when needed:

(H1) There exist some $s_{1} \in(0,1]$ and a constant $L_{1} \geq 0$ such that for all $u \in V$,

$$
\|g(u)\|_{*} \leq L_{1}(1+|u|)^{s_{1}}\|u\|^{1-s_{1}} .
$$

(H2) There exists some $s_{2} \in(0,1]$ such that for every $M>0$ there is a constant $L_{2}=L_{2}(M) \geq 0$ and for all $u, v \in \mathcal{B}_{M}$,

$$
\|g(u)-g(v)\|_{*} \leq L_{2}|u-v|^{s_{2}}\|u-v\|^{1-s_{2}} .
$$

(H3) The function $g$ is weakly sequentially continuous from $V$ into $V^{*}$, i.e., $u_{m} \rightarrow u$ in $V$ implies $g\left(u_{m}\right) \rightarrow g(u)$ in $V^{*}$ as $m \rightarrow \infty$.

We remark that $(\mathrm{H} 1)$ includes (with $s_{1}=1$ ) the case $\|g(u)\|_{*} \leq$ const. Note that the constant $L_{2}$ in the Lipschitz-like condition (H2) may increase with the radius $M$. Furthermore, if the embedding $V \hookrightarrow H$ is compact then (H2) implies strong continuity of $g: V \rightarrow V^{*}$ and hence (H3). Instead of (H1), (H2), and (H3), we may, alternatively, assume $g(V) \subseteq H$ and the following: 
$\widetilde{(\mathrm{H} 1)}$ There exists a constant $\widetilde{L}_{1} \geq 0$ such that for all $u \in V$,

$$
|g(u)| \leq \widetilde{L}_{1}(1+\|u\|) .
$$

$\widetilde{(\mathrm{H} 2)}$ For every $M>0$ there is a constant $\widetilde{L}_{2}=\widetilde{L}_{2}(M) \geq 0$ such that for all $u, v \in \mathcal{B}_{M}$

$$
|g(u)-g(v)| \leq \widetilde{L}_{2}\|u-v\| .
$$

(H3) In addition to (H3), there holds: $u_{m} \rightarrow u$ in $L^{2}(0, T ; V)$ and $u_{m} \rightarrow u$ in $L^{2}(0, T ; H)$ implies $g\left(u_{m}\right) \rightarrow g(u)$ in $L^{2}(0, T ; H)$ as $m \rightarrow \infty$.

Note again that $\tilde{L}_{2}$ may depend on the radius $M$.

For a Banach space $X$ and a time interval $S \subseteq \mathbb{R}$, let $L^{p}(S ; X)(p \in[1, \infty])$ be the usual spaces of Bochner integrable functions. The discrete counterparts for functions defined on a time grid are denoted by $l^{p}(0, T ; X)$. Note that $u \in$ $L^{2}(0, T ; V), u^{\prime} \in L^{2}\left(0, T ; V^{*}\right)$ implies $u \in \mathcal{C}([0, T] ; H)$ in the sense that $u$ is almost everywhere in $S$ equal to an abstract function that is continuous on $[0, T]$ with respect to the strong convergence in $H$. With $u^{\prime}$, the derivative in the distributional sense is meant.

The problem we are concerned with then reads as

Problem $(P)$ For given $u_{0} \in H$ and $f \in L^{2}\left(0, T ; V^{*}\right)$, find $u \in L^{2}(0, T ; V)$ with $u^{\prime} \in L^{2}\left(0, T ; V^{*}\right)$ such that for all $v \in V$ and almost everywhere in $(0, T)$

$$
\frac{d}{d t}(u(t), v)+a(u(t), v)+\langle g(u(t)), v\rangle=\langle f(t), v\rangle
$$

holds with $u(0)=u_{0}$.

Note that $\left\langle u^{\prime}(t), v\right\rangle=\frac{d}{d t}(u(t), v)$ holds for all $u \in L^{2}(0, T ; V)$ with $u^{\prime} \in$ $L^{2}\left(0, T ; V^{*}\right)$ and all $v \in V$ in the distributional sense on $(0, T)$.

For what we have in mind, we give the following

\section{Standard example (Dirichlet problem for a second order PDE).}

Let $V=H_{0}^{1}(\Omega)$ and $H=L^{2}(\Omega)$ be the usual Sobolev and Lebesgue space, respectively, for some bounded domain $\Omega \subset \mathbb{R}^{d}(d \in \mathbb{N})$ with sufficiently smooth boundary. The bilinear form $a(\cdot, \cdot)$ is defined by

$$
a(u, v):=\int_{\Omega}\left(\sum_{i, j=1}^{d} a_{i j} \frac{\partial u}{\partial x_{i}} \frac{\partial v}{\partial x_{j}}+\sum_{i=1}^{d} b_{i} \frac{\partial u}{\partial x_{i}} v+c u v\right) d x,
$$

where $a_{i j}, b_{i}, c$ are sufficiently smooth functions in $x \in \Omega$ with $\left(a_{i j}\right)$ being symmetric and uniformly positive definite and with

$$
\underset{x \in \Omega}{\operatorname{ess} \sup _{0}}\left(c(x)-\frac{1}{2} \sum_{i=1}^{d} \frac{\partial b_{i}(x)}{\partial x_{i}}\right) \geq 0 .
$$


The nonlinearity might be given by a Lipschitz-continuous real function $g=g(s)$ with at most linear growth,

$$
|g(s)| \leq c(1+|s|), \quad s \in \mathbb{R},
$$

for some constant $c$, although more complicated nonlinearities are allowed. In virtue of the Poincaré-Friedrichs inequality, the structural assumptions $\widetilde{(\mathrm{H} 1)}$ and $\widetilde{(\mathrm{H} 2)}$ are fulfilled. Because of the compact embedding of $V$ into $H$ and the Lipschitz continuity, $g$ becomes a strongly continuous mapping from $V$ into $H$ and is continuous from $L^{2}(0, T ; H)$ into $L^{2}(0, T ; H)$. Thus $\widetilde{(\mathrm{H} 3)}$ is fulfilled.

We shall remark that a bilinear form that only satisfies a Gårding inequality can be also treated by collecting the disturbing linear terms into $g$, and so condition (2.5) is not important. Let us finally note that $V$ and $H$ can be finite dimensional as is the case when first discretising in space and afterwards in time. For the spatial approximation, a conforming finite element method might be used. For more details, we refer to Thomée [20] and the references cited therein.

With respect to the solvability of Problem $(P)$, we have

Theorem 1. Problem $(P)$ admits a unique solution $u \in L^{2}(0, T ; V) \cap \mathcal{C}([0, T]$; $H)$ with $u^{\prime} \in L^{2}\left(0, T ; V^{*}\right)$ if $(\mathrm{H} 1),(\mathrm{H} 2)$, and (H3) or, alternatively, $\widetilde{(\mathrm{H} 1)}, \widetilde{(\mathrm{H} 2)}$, and $\widetilde{(\mathrm{H} 3)}$ hold true.

A proof will be given in Appendix B. The time discretisation of Problem $(P)$ reads as

Problem $\left(P_{\tau}\right)$. For given $u^{0}, u^{1} \in H$ and $\left\{f^{n}\right\} \in l^{2}\left(0, T ; V^{*}\right)$, find $u^{n} \in V$ $(n=2,3, \ldots, N)$ such that for all $v \in V$,

$$
\left(\mathrm{D}_{2} u^{n}, v\right)+a\left(u^{n}, v\right)+\left\langle g\left(u^{n}\right), v\right\rangle=\left\langle f^{n}, v\right\rangle .
$$

We may suppose $u^{0}=u_{0}$ and compute $u^{1}$ by the implicit Euler method, although our results do not require a particular method for the first step. With respect to the solvability of Problem $\left(P_{\tau}\right)$, we have

Theorem 2. Assume (H1), (H2) or, alternatively, $\widetilde{(\mathrm{H} 1)}, \widetilde{(\mathrm{H} 2})$, and $(\mathrm{H} 3)$. Problem $\left(P_{\tau}\right)$ admits a unique solution if $\tau_{\max }:=\max _{n=1, \ldots, N} \tau_{n}$ is sufficiently small.

In the linear case, there is no restriction on $\tau_{\max }$. A proof is provided in Appendix B. 


\section{Stability and error in the linear case}

In the following, let $c>0$ be a generic constant that does not depend on problem parameters (except $\bar{R}$ ) whereas $C>0$ may depend on $T, R$, the sequence of step size ratios as well as on constants that appear in the assumptions on $a(\cdot, \cdot)$ and $g(\cdot)$. Note the conventions $\sum_{j=m}^{n} x_{j}:=0$ and $\prod_{j=m}^{n} x_{j}:=1$ if $m>n$.

Theorem 3. If $R<\bar{R}$, the solution to Problem $\left(P_{\tau}\right)$ with $g(u) \equiv 0$ is stable in $l^{\infty}(0, T ; H)$ and $l^{2}(0, T ; V)$. The following estimate holds for $n=2,3, \ldots, N$ :

$$
\left|u^{n}\right|^{2}+\sum_{j=2}^{n} \tau_{j}\left\|u^{j}\right\|^{2} \leq C\left(\left|u^{0}\right|^{2}+\left|u^{1}\right|^{2}+\tau_{2}\left\|u^{1}\right\|^{2}+\sum_{j=2}^{n} \frac{\tau_{j}}{1+r_{j}}\left\|f^{j}\right\|_{*}^{2}\right) .
$$

Proof. The proof follows mainly the ideas of Becker [1]. We test (2.6) with

$$
u_{\delta}^{n}:=u^{n}+\delta \tau_{n} \mathrm{D}_{1} u^{n}=(1+\delta) u^{n}-\delta u^{n-1}
$$

for some $\delta>0$ specified later and multiply by $2 \tau_{n} /\left(1+r_{n}\right)$. The proof then consists of three steps:

i) estimation of $2 \tau_{n}\left(\mathrm{D}_{2} u^{n}, u_{\delta}^{n}\right) /\left(1+r_{n}\right)$,

ii) estimation of $2 \tau_{n} a\left(u^{n}, u_{\delta}^{n}\right) /\left(1+r_{n}\right)$ and $2 \tau_{n}\left\langle f^{n}, u_{\delta}^{n}\right\rangle /\left(1+r_{n}\right)$, and

iii) final derivation of the assertion.

ad i). We firstly observe by simple calculations and Young's inequality that

$$
\begin{aligned}
& \frac{2 \tau_{n}}{1+r_{n}}\left(\mathrm{D}_{2} u^{n}, u_{\delta}^{n}\right) \\
= & \frac{\tau_{n}}{1+r_{n}} \mathrm{D}_{2}\left|u^{n}\right|^{2}+(1+2 \delta) \frac{1+2 r_{n}}{\left(1+r_{n}\right)^{2}}\left|\tau_{n} \mathrm{D}_{1} u^{n}\right|^{2} \\
& \quad-\frac{r_{n}^{2}}{\left(1+r_{n}\right)^{2}}\left|\tau_{n-1} \mathrm{D}_{1} u^{n-1}\right|^{2}-2(1+\delta) \frac{r_{n}^{2}}{\left(1+r_{n}\right)^{2}}\left(\tau_{n} \mathrm{D}_{1} u^{n}, \tau_{n-1} \mathrm{D}_{1} u^{n-1}\right) \\
\geq & \frac{\tau_{n}}{1+r_{n}} \mathrm{D}_{2}\left|u^{n}\right|^{2}+A_{\delta}\left(r_{n}\right)\left|\tau_{n} \mathrm{D}_{1} u^{n}\right|^{2}-B_{\delta}\left(r_{n}\right)\left|\tau_{n-1} \mathrm{D}_{1} u^{n-1}\right|^{2},
\end{aligned}
$$

where

$$
A_{\delta}\left(r_{n}\right):=\frac{1+2 r_{n}-r_{n}^{2}+\delta\left(2+4 r_{n}-r_{n}^{2}\right)}{\left(1+r_{n}\right)^{2}}, \quad B_{\delta}\left(r_{n}\right):=(2+\delta) \frac{r_{n}^{2}}{\left(1+r_{n}\right)^{2}} .
$$


Summation gives

$$
\begin{aligned}
2 \sum_{j=2}^{n} \frac{\tau_{j}}{1+r_{j}} & \left(\mathrm{D}_{2} u^{j}, u_{\delta}^{j}\right) \geq \sum_{j=2}^{n} \frac{\tau_{j}}{1+r_{j}} \mathrm{D}_{2}\left|u^{j}\right|^{2}+A_{\delta}\left(r_{n}\right)\left|\tau_{n} \mathrm{D}_{1} u^{n}\right|^{2} \\
& +\sum_{j=2}^{n-1}\left(A_{\delta}\left(r_{j}\right)-B_{\delta}\left(r_{j+1}\right)\right)\left|\tau_{j} \mathrm{D}_{1} u^{j}\right|^{2}-B_{\delta}\left(r_{2}\right)\left|\tau_{1} \mathrm{D}_{1} u^{1}\right|^{2}
\end{aligned}
$$

Since $r \mapsto A_{\delta}(r)$ is decreasing whereas $r \mapsto B_{\delta}(r)$ is increasing, we find

$$
A_{\delta}\left(r_{j}\right)-B_{\delta}\left(r_{j+1}\right) \geq A_{\delta}(R)-B_{\delta}(R)=\frac{1+2 R-3 R^{2}+2 \delta\left(1+2 R-R^{2}\right)}{(1+R)^{2}} .
$$

For $A_{\delta}\left(r_{j}\right)-B_{\delta}\left(r_{j+1}\right) \geq 0$ to hold, $\delta$ is taken as

$$
\hat{\delta}=\hat{\delta}(R)=-\frac{1}{2} \frac{1+2 R-3 R^{2}}{1+2 R-R^{2}}=-\frac{3(R-1)\left(R+\frac{1}{3}\right)}{2(R-1-\sqrt{2})(R-1+\sqrt{2})},
$$

taking into account that $1<R<1+\sqrt{2}$. We then have for $1<R<\bar{R}$ that

$$
\frac{1}{2}<A_{\hat{\delta}}(R)=B_{\hat{\delta}}(R)=\frac{R^{2}(R-3-2 \sqrt{3})(R-3+2 \sqrt{3})}{2(1+R)^{2}(R-1-\sqrt{2})(R-1+\sqrt{2})}<2 .
$$

For the first term of the right-hand side of (3.2), we have

$$
\begin{aligned}
\sum_{j=2}^{n} \frac{\tau_{j}}{1+r_{j}} \mathrm{D}_{2}\left|u^{j}\right|^{2}= & \sum_{j=2}^{n}\left(\frac{1+2 r_{j}}{\left(1+r_{j}\right)^{2}}\left|u^{j}\right|^{2}-\left|u^{j-1}\right|^{2}+\frac{r_{j}^{2}}{\left(1+r_{j}\right)^{2}}\left|u^{j-2}\right|^{2}\right) \\
= & \frac{1+2 r_{n}}{\left(1+r_{n}\right)^{2}}\left|u^{n}\right|^{2}-\frac{r_{n-1}^{2}}{\left(1+r_{n-1}\right)^{2}}\left|u^{n-1}\right|^{2} \\
& +\sum_{j=2}^{n-2}\left(\frac{1+2 r_{j}}{\left(1+r_{j}\right)^{2}}-1+\frac{r_{j+2}^{2}}{\left(1+r_{j+2}\right)^{2}}\right)\left|u^{j}\right|^{2} \\
& -\frac{1+2 r_{3}}{\left(1+r_{3}\right)^{2}}\left|u^{1}\right|^{2}+\frac{r_{2}^{2}}{\left(1+r_{2}\right)^{2}}\left|u^{0}\right|^{2} .
\end{aligned}
$$

Furthermore, there holds for some $\bar{r}$ between $r_{j}$ and $r_{j+2}$

$$
\begin{aligned}
& \frac{1+2 r_{j}}{\left(1+r_{j}\right)^{2}}- 1+\frac{r_{j+2}^{2}}{\left(1+r_{j+2}\right)^{2}}=\frac{r_{j+2}^{2}}{\left(1+r_{j+2}\right)^{2}}-\frac{r_{j}^{2}}{\left(1+r_{j}\right)^{2}} \\
&=\frac{d}{d r}\left(\left(\frac{r}{1+r}\right)^{2}\right)_{\mid r=\bar{r}}\left(r_{j+2}-r_{j}\right) \geq-\frac{8}{27}\left[r_{j+2}-r_{j}\right]_{-} .
\end{aligned}
$$

This lower bound follows since the nonnegative function $r \mapsto \frac{d}{d r}\left(r^{2} /(1+r)^{2}\right)$ takes its maximum value $8 / 27$ at $r=1 / 2$. Using monotonicity arguments, we 
finally obtain from (3.2), (3.3), and (3.4)

$$
\begin{aligned}
2 \sum_{j=2}^{n} \frac{\tau_{j}}{1+r_{j}} & \left(\mathrm{D}_{2} u^{j}, u_{\hat{\delta}}^{j}\right) \geq \frac{1+2 R}{(1+R)^{2}}\left|u^{n}\right|^{2}-\frac{R^{2}}{(1+R)^{2}}\left|u^{n-1}\right|^{2}+\frac{1}{2}\left|u^{n}-u^{n-1}\right|^{2} \\
& -\left(\left|u^{1}\right|^{2}+2\left|u^{1}-u^{0}\right|^{2}+\frac{8}{27} \sum_{j=2}^{n-2}\left[r_{j+2}-r_{j}\right]_{-}\left|u^{j}\right|^{2}\right)
\end{aligned}
$$

which makes sense since $1+2 R>R^{2}$ as $1<R<1+\sqrt{2}$.

ad ii). We may prove the identity

$$
\begin{gathered}
2 a\left(u^{n}, u_{\hat{\delta}}^{n}\right)=\frac{1}{1+\hat{\delta}} a\left(u_{\hat{\delta}}^{n}, u_{\hat{\delta}}^{n}\right)+(1+\hat{\delta}) a\left(u^{n}, u^{n}\right)-\frac{\hat{\delta}^{2}}{1+\hat{\delta}} a\left(u^{n-1}, u^{n-1}\right) \\
-\frac{\hat{\delta}}{1+\hat{\delta}}\left(a\left(u_{\hat{\delta}}^{n}, u^{n-1}\right)-a\left(u^{n-1}, u_{\hat{\delta}}^{n}\right)\right) .
\end{gathered}
$$

With (2.3), (2.2), and Young's inequality, we find

$$
\hat{\delta}\left|a\left(u_{\hat{\delta}}^{n}, u^{n-1}\right)-a\left(u^{n-1}, u_{\hat{\delta}}^{n}\right)\right| \leq \hat{\delta} \gamma\left\|u_{\hat{\delta}}^{n}\right\|\left|u^{n-1}\right| \leq \frac{1}{2} a\left(u_{\hat{\delta}}^{n}, u_{\hat{\delta}}^{n}\right)+\frac{\hat{\delta}^{2} \gamma^{2}}{2 \mu}\left|u^{n-1}\right|^{2} .
$$

So it follows from (3.6) that

$$
\begin{gathered}
2 a\left(u^{n}, u_{\hat{\delta}}^{n}\right) \geq \frac{1}{2(1+\hat{\delta})} a\left(u_{\hat{\delta}}^{n}, u_{\hat{\delta}}^{n}\right)+(1+\hat{\delta}) a\left(u^{n}, u^{n}\right)-\frac{\hat{\delta}^{2}}{1+\hat{\delta}} a\left(u^{n-1}, u^{n-1}\right) \\
-\frac{\hat{\delta}^{2} \gamma^{2}}{2 \mu(1+\hat{\delta})}\left|u^{n-1}\right|^{2} .
\end{gathered}
$$

Furthermore, we have with (2.2) and Young's inequality

$$
2\left\langle f^{n}, u_{\hat{\delta}}^{n}\right\rangle \leq 2\left\|f^{n}\right\|_{*}\left\|u_{\hat{\delta}}^{n}\right\| \leq \frac{2(1+\hat{\delta})}{\mu}\left\|f^{n}\right\|_{*}^{2}+\frac{1}{2(1+\hat{\delta})} a\left(u_{\hat{\delta}}^{n}, u_{\hat{\delta}}^{n}\right) ;
$$

the term $a\left(u_{\hat{\delta}}^{n}, u_{\hat{\delta}}^{n}\right) /(2(1+\hat{\delta}))$ will be absorbed within (3.7).

With (3.5), (3.7), and (3.8), we now obtain

$$
\begin{gathered}
\frac{1+2 R}{(1+R)^{2}}\left|u^{n}\right|^{2}-\frac{R^{2}}{(1+R)^{2}}\left|u^{n-1}\right|^{2}+\frac{1}{2}\left|u^{n}-u^{n-1}\right|^{2}+\frac{\tau_{n}(1+\hat{\delta})}{1+r_{n}} a\left(u^{n}, u^{n}\right) \\
+\sum_{j=2}^{n-1} \frac{\tau_{j}}{1+r_{j}}\left(1+\hat{\delta}-\frac{\hat{\delta}^{2}}{1+\hat{\delta}} \frac{r_{j+1}\left(1+r_{j}\right)}{1+r_{j+1}}\right) a\left(u^{j}, u^{j}\right) \\
\leq\left|u^{1}\right|^{2}+2\left|u^{1}-u^{0}\right|^{2}+\frac{\hat{\delta}^{2}}{1+\hat{\delta}} \frac{\tau_{2}}{1+r_{2}} a\left(u^{1}, u^{1}\right)+\frac{8}{27} \sum_{j=2}^{n-2}\left[r_{j+2}-r_{j}\right]_{-}\left|u^{j}\right|^{2}
\end{gathered}
$$




$$
+\frac{\hat{\delta}^{2} \gamma^{2}}{2 \mu(1+\hat{\delta})} \sum_{j=2}^{n} \frac{\tau_{j}}{1+r_{j}}\left|u^{j-1}\right|^{2}+\frac{2(1+\hat{\delta})}{\mu} \sum_{j=2}^{n} \frac{\tau_{j}}{1+r_{j}}\left\|f^{j}\right\|_{*}^{2} .
$$

ad iii). Since $r_{j+1}\left(1+r_{j}\right) /\left(1+r_{j+1}\right) \leq R$, we need that $1+\hat{\delta}>R \hat{\delta}^{2} /(1+\hat{\delta})$, i.e.,

$$
R<\left(\frac{1+\hat{\delta}}{\hat{\delta}}\right)^{2}=\frac{(R+1)^{4}}{9(R-1)^{2}\left(R+\frac{1}{3}\right)^{2}},
$$

in order to prove the stability result. Unfortunately, relation (3.10), which is equivalent to $\psi(R)>0$ (see (1.1)), is only satisfied for $R<\bar{R}$. With

$$
\sigma(R):=\frac{1}{1+R}\left(1+\hat{\delta}-R \frac{\hat{\delta}^{2}}{1+\hat{\delta}}\right)=\frac{\psi(R)}{2(1+R)^{3}\left(1+2 R-R^{2}\right)}>0,
$$

using $1<R<\bar{R}$, and in virtue of (3.9), we come up with

$$
\begin{gathered}
\frac{1+2 R}{(1+R)^{2}}\left|u^{n}\right|^{2}+\frac{1}{2}\left|u^{n}-u^{n-1}\right|^{2}+\frac{1+\hat{\delta}}{1+R} \mu \tau_{n}\left\|u^{n}\right\|^{2}+\mu \sigma(R) \sum_{j=2}^{n-1} \tau_{j}\left\|u^{j}\right\|^{2} \\
\leq \frac{R^{2}}{(1+R)^{2}}\left|u^{n-1}\right|^{2}+K_{n}
\end{gathered}
$$

where

$$
\begin{aligned}
K_{n}:=c\left(\sum _ { j = 2 } ^ { n - 2 } \left[r_{j+2}\right.\right. & \left.-r_{j}\right]_{-}\left|u^{j}\right|^{2}+\frac{\gamma^{2}}{\mu} \sum_{j=2}^{n-1} \tau_{j+1}\left|u^{j}\right|^{2} \\
& \left.+\left|u^{0}\right|^{2}+\left|u^{1}\right|^{2}+\beta \tau_{2}\left\|u^{1}\right\|^{2}+\frac{1}{\mu} \sum_{j=2}^{n} \frac{\tau_{j}}{1+r_{j}}\left\|f^{j}\right\|_{*}^{2}\right) .
\end{aligned}
$$

Let $m^{*}=m^{*}(m)$ be such that $\left|u^{m^{*}}\right|=\max _{l=1, \ldots, m}\left|u^{l}\right|$ for $m=2,3, \ldots, N$. If $m^{*} \geq 2$, it follows from (3.11) with $n=m^{*}$ and because of $K_{m^{*}} \leq K_{m}$ the estimate

$$
\frac{1+2 R}{(1+R)^{2}}\left|u^{m^{*}}\right|^{2} \leq \frac{R^{2}}{(1+R)^{2}}\left|u^{m^{*}-1}\right|^{2}+K_{m^{*}} \leq \frac{R^{2}}{(1+R)^{2}}\left|u^{m^{*}}\right|^{2}+K_{m}
$$

that leads for $R<1+\sqrt{2}$ to

$$
\left|u^{m^{*}}\right|^{2} \leq \frac{(1+R)^{2}}{1+2 R-R^{2}} K_{m} .
$$

This last estimate holds also true if $m^{*}=1$. With $n=m$ in (3.11) and $\left|u^{m-1}\right| \leq$ $\left|u^{m^{*}}\right|$, we thus have (changing $m, n$ again) for $n=2,3, \ldots, N$

$$
\frac{1+2 R}{(1+R)^{2}}\left|u^{n}\right|^{2}+\frac{1}{2}\left|u^{n}-u^{n-1}\right|^{2}+\frac{1+\hat{\delta}}{1+R} \mu \tau_{n}\left\|u^{n}\right\|^{2}+\mu \sigma(R) \sum_{j=2}^{n-1} \tau_{j}\left\|u^{j}\right\|^{2}
$$




$$
\leq\left(\frac{R^{2}}{1+2 R-R^{2}}+1\right) K_{n}=\frac{1+2 R}{1+2 R-R^{2}} K_{n} .
$$

Taking into account that $1<R<\bar{R} \approx 1.91$ yields

$$
\left|u^{n}\right|^{2}+\left|u^{n}-u^{n-1}\right|^{2}+\mu \tau_{n}\left\|u^{n}\right\|^{2}+\mu \psi(R) \sum_{j=2}^{n-1} \tau_{j}\left\|u^{j}\right\|^{2} \leq c K_{n} .
$$

It follows from Lemma 1 in Appendix A that

$$
\begin{aligned}
& \left|u^{n}\right|^{2}+\left|u^{n}-u^{n-1}\right|^{2}+\mu \tau_{n}\left\|u^{n}\right\|^{2}+\mu \psi(R) \sum_{j=2}^{n-1} \tau_{j}\left\|u^{j}\right\|^{2} \\
& \leq c \Lambda_{n}\left(\left|u^{0}\right|^{2}+\left|u^{1}\right|^{2}+\beta \tau_{2}\left\|u^{1}\right\|^{2}+\frac{1}{\mu} \sum_{j=2}^{n} \frac{\tau_{j}}{1+r_{j}}\left\|f^{j}\right\|^{2}\right),
\end{aligned}
$$

where

$$
\Lambda_{n}=\left(1+\frac{c \gamma^{2} \tau_{n}}{\mu}\right) \prod_{j=2}^{n-2}\left(1+c\left[r_{j+2}-r_{j}\right]_{-}+\frac{c \gamma^{2} \tau_{j+1}}{\mu}\right) \leq \exp \left(c \Gamma_{n}+\frac{c \gamma^{2} t_{n}}{\mu}\right)
$$

with $\Gamma_{n}=\sum_{j=2}^{n-2}\left[r_{j+2}-r_{j}\right]_{-}(n=2, \ldots, N)$. This proves the assertion.

We shall remark that Becker's proof (cf. [1]) relies upon the identity

$$
\begin{gathered}
2 a\left(u^{n}, u_{\hat{\delta}}^{n}\right)=\hat{\delta} a\left(u^{n}-u^{n-1}, u^{n}-u^{n-1}\right)+(2+\hat{\delta}) a\left(u^{n}, u^{n}\right)-\hat{\delta} a\left(u^{n-1}, u^{n-1}\right) \\
-\hat{\delta}\left(a\left(u^{n}, u^{n-1}\right)-a\left(u^{n-1}, u^{n}\right)\right)
\end{gathered}
$$

instead of (3.6), which finally leads to the condition $2+\hat{\delta}>R \hat{\delta}$ instead of (3.10). This condition is fulfilled if $R<(2+\sqrt{13}) / 2 \approx 1.868<\bar{R} \approx 1.910$.

Remark 1. With the natural restriction

$$
\mathrm{R}_{2}^{j} f:=\left(1+\frac{r_{j}}{1+r_{j}}\right) \mathrm{R}_{1}^{j} f-\frac{r_{j}}{1+r_{j}} \mathrm{R}_{1}^{j-1} f, \quad \mathrm{R}_{1}^{j} f:=\frac{1}{\tau_{j}} \int_{t_{j-1}}^{t_{j}} f(t) d t,
$$

we obtain for $f^{j}=\mathrm{R}_{2}^{j} f$ by standard arguments that

$$
\sum_{j=2}^{n} \frac{\tau_{j}}{1+r_{j}}\left\|f^{j}\right\|_{*}^{2} \leq c \int_{0}^{t_{n}}\|f(t)\|_{*}^{2} d t, \quad n=2, \ldots, N .
$$

Note that $\mathrm{R}_{1}^{j} u^{\prime}=\mathrm{D}_{1} u\left(t_{j}\right)$ and $\mathrm{R}_{2}^{j} u^{\prime}=\mathrm{D}_{2} u\left(t_{j}\right)$ for sufficiently smooth $u$. 
The constant $C$ in (3.1) is quantified through (3.13) and (3.14).

Theorem 4. Let $f^{n}=\mathrm{R}_{2}^{n} f$ and $f^{\prime}-u^{\prime \prime}, f^{\prime \prime}-u^{\prime \prime \prime} \in L^{2}\left(0, T ; V^{*}\right)$. If $R<\bar{R}$ then the error $e^{n}=u\left(t_{n}\right)-u^{n}(n=2,3, \ldots, N)$ to Problem $\left(P_{\tau}\right)$ with $g(u) \equiv 0$ satisfies

$$
\begin{gathered}
\left|e^{n}\right|^{2}+\sum_{j=2}^{n} \tau_{j}\left\|e^{j}\right\|^{2} \leq C\left(\left|e^{0}\right|^{2}+\left|e^{1}\right|^{2}+\tau_{2}\left\|e^{1}\right\|^{2}\right. \\
+\sum_{j=2}^{n}\left(\operatorname{sgn}\left(r_{j}\right)\left(\tau_{j}^{4} \int_{t_{j-1}}^{t_{j}}\left\|f^{\prime \prime}(t)-u^{\prime \prime \prime}(t)\right\|_{*}^{2} d t+\tau_{j-1}^{4} \int_{t_{j-2}}^{t_{j-1}}\left\|f^{\prime \prime}(t)-u^{\prime \prime \prime}(t)\right\|_{*}^{2} d t\right)\right. \\
\left.\left.+\left(1-\operatorname{sgn}\left(r_{j}\right)\right) \tau_{j}^{2} \int_{t_{j-1}}^{t_{j}}\left\|f^{\prime}(t)-u^{\prime \prime}(t)\right\|_{*}^{2} d t\right)\right)
\end{gathered}
$$

Proof. We commence with the corresponding error equation

$$
\left(\mathrm{D}_{2} e^{n}, v\right)+a\left(e^{n}, v\right)=\left\langle\rho^{n}, v\right\rangle \quad \forall v \in V
$$

that follows from (2.4) and (2.6) with the consistency error

$$
\rho^{n}=\mathrm{D}_{2} u\left(t_{n}\right)-u^{\prime}\left(t_{n}\right)+f\left(t_{n}\right)-\mathrm{R}_{2}^{n} f=\mathrm{I}_{2}^{n}\left(f^{\prime \prime}-u^{\prime \prime \prime}\right),
$$

where

$$
\begin{aligned}
\mathrm{I}_{2}^{n} w:=\frac{1}{2\left(1+r_{n}\right)}\left(\frac{1}{\tau_{n}} \int_{t_{n-1}}^{t_{n}}\left(t_{n}-t\right)\left(\left(1+2 r_{n}\right)\left(t-t_{n-1}\right)+\tau_{n}\right) w(t) d t\right. \\
\left.+\frac{r_{n}}{\tau_{n-1}} \int_{t_{n-2}}^{t_{n-1}}\left(t-t_{n-2}\right)^{2} w(t) d t\right) .
\end{aligned}
$$

If $r_{n}=0$ (occasional Euler step), then $\mathrm{D}_{2}$ and $\mathrm{R}_{2}$ degenerate to $\mathrm{D}_{1}$ and $\mathrm{R}_{1}$ respectively, and we come up with

$$
\rho^{n}=\mathrm{D}_{1} u\left(t_{n}\right)-u^{\prime}\left(t_{n}\right)+f\left(t_{n}\right)-\mathrm{R}_{1}^{n} f=\mathrm{I}_{1}^{n}\left(f^{\prime}-u^{\prime \prime}\right),
$$

where

$$
\mathrm{I}_{1}^{n} w:=\frac{1}{\tau_{n}} \int_{t_{n-1}}^{t_{n}}\left(t-t_{n-1}\right) w(t) d t .
$$

The assertion follows from Theorem 3 because of

$$
\frac{\tau_{j}}{1+r_{j}}\left\|\mathrm{I}_{2}^{j} w\right\|_{*}^{2} \leq c \tau_{j}^{4} \int_{t_{j-1}}^{t_{j}}\|w(t)\|_{*}^{2} d t+c \tau_{j-1}^{4} \int_{t_{j-2}}^{t_{j-1}}\|w(t)\|_{*}^{2} d t
$$

and

$$
\frac{\tau_{j}}{1+r_{j}}\left\|\mathrm{I}_{1}^{j} w\right\|_{*}^{2} \leq c \tau_{j}^{2} \int_{t_{j-1}}^{t_{j}}\|w(t)\|_{*}^{2} d t
$$


Remark 2. As one can see from estimate (3.15), it suffices to start with any first order scheme, and even during the computation, an occasional change to the implicit Euler scheme $\left(r_{n}=0\right)$ of at most a fixed number of times does not affect the second order convergence. Those changes might be useful as they give some stabilisation. Estimate (3.15) also reflects the lower order of the implicit Euler method.

A more precise estimate is again given by (3.13) and (3.14), replacing $u^{n}$ by $e^{n}$ and $f^{n}$ by $\rho^{n}$. This shows the dependence of the error constant on problem parameters. The use of the natural restriction is only for simplicity. Otherwise, there appears the additional error term $\sum_{j} \tau_{j}\left\|f^{j}-\mathrm{R}_{2}^{j} f\right\|_{*}^{2}$. The regularity assumptions $f^{\prime}-u^{\prime \prime}, f^{\prime \prime}-u^{\prime \prime \prime} \in L^{2}\left(0, T ; V^{*}\right)$ are fulfilled if e. g. $A^{2} u_{0} \in H$ and $A f, f^{\prime} \in L^{2}(0, T ; V)$ or if $f, f^{\prime}, f^{\prime \prime} \in L^{2}\left(0, T ; V^{*}\right)$ and $u_{0}, f(0)-A u_{0} \in V$, $f^{\prime}(0)+A f(0)+A^{2} u_{0} \in H$.

\section{Stability and error in the nonlinear case}

Theorem 5. Under the assumption (H1) (or, alternatively, $\widetilde{(\mathrm{H} 1)}$ ), the solution to Problem $\left(P_{\tau}\right)$ is stable in $l^{\infty}(0, T ; H)$ and $l^{2}(0, T ; V)$ if $R<\bar{R}$ and $\tau_{\max }$ is sufficiently small. The following estimate holds for $n=2,3, \ldots, N$ :

$$
\left|u^{n}\right|^{2}+\sum_{j=2}^{n} \tau_{j}\left\|u^{j}\right\|^{2} \leq C\left(\left|u^{0}\right|^{2}+\left|u^{1}\right|^{2}+\tau_{2}\left\|u^{1}\right\|^{2}+\sum_{j=2}^{n} \frac{\tau_{j}}{1+r_{j}}\left\|f^{j}\right\|_{*}^{2}+t_{n}\right) .
$$

Proof. We reconsider the proof of Theorem 3. For the term $\left\langle g\left(u^{n}\right), u_{\hat{\delta}}^{n}\right\rangle$, which describes the nonlinearity, we obtain from (H1) for arbitrary $\varepsilon \in(0,1)$ with the Cauchy-Schwarz and Young inequality

$$
\begin{gathered}
2\left|\left\langle g\left(u^{n}\right), u_{\hat{\delta}}^{n}\right\rangle\right| \leq 2\left\|g\left(u^{n}\right)\right\|_{*}\left\|u_{\hat{\delta}}^{n}\right\| \leq\left. 2 L_{1}\left(1+\left|u^{n}\right|\right)^{s_{1}}\left\|u^{n}\right\|\right|^{1-s_{1}}\left\|u_{\hat{\delta}}^{n}\right\| \\
\leq C_{1}\left(1+\left|u^{n}\right|^{2}\right)+\varepsilon a\left(u^{n}, u^{n}\right)+\frac{\varepsilon}{2(1+\hat{\delta})} a\left(u_{\hat{\delta}}^{n}, u_{\hat{\delta}}^{n}\right),
\end{gathered}
$$

where

$$
C_{1}=\left\{\begin{array}{cl}
\frac{2 \varepsilon \mu s_{1}}{1-s_{1}}\left(\frac{2\left(1-s_{1}\right)(1+\hat{\delta}) L_{1}^{2}}{\varepsilon^{2} \mu^{2}}\right)^{1 / s_{1}} & \text { if } s_{1} \neq 1, \\
\frac{4(1+\hat{\delta}) L_{1}^{2}}{\varepsilon \mu} & \text { if } s_{1}=1 .
\end{array}\right.
$$

Changing the coefficients in the right-hand side of (3.8) appropriately such that

$$
2\left\langle f^{n}, u_{\hat{\delta}}^{n}\right\rangle \leq \frac{2(1+\hat{\delta})}{(1-\varepsilon) \mu}\left\|f^{n}\right\|_{*}^{2}+\frac{1-\varepsilon}{2(1+\hat{\delta})} a\left(u_{\hat{\delta}}^{n}, u_{\hat{\delta}}^{n}\right)
$$


the terms with $a\left(u_{\hat{\delta}}^{n}, u_{\hat{\delta}}^{n}\right)$ can be absorbed in view of (3.7). The additional term $\varepsilon a\left(u^{n}, u^{n}\right)$ (which does not appear if $s_{1}=1$ ) requires, however, to modify the crucial condition (3.10) that came from (3.9). Here, we need that $1+\hat{\delta}-\varepsilon>$ $R \hat{\delta}^{2} /(1+\hat{\delta})$. For any $R<\bar{R}$, this can be fulfilled by taking $\varepsilon$ sufficiently small. The remaining term $C_{1}\left(1+\left|u^{n}\right|^{2}\right)$ leads to a change of the right-hand sides in (3.11) and the following estimates: We take

$$
\begin{gathered}
K_{n}=c\left(\sum_{j=2}^{n-2}\left[r_{j+2}-r_{j}\right]_{-}\left|u^{j}\right|^{2}+\sum_{j=2}^{n-1}\left(C_{1} \tau_{j}+\frac{\gamma^{2} \tau_{j+1}}{\mu}\right)\left|u^{j}\right|^{2}+C_{1} \tau_{\max }\left|u^{n}\right|^{2}\right. \\
\left.\quad+C_{1} t_{n}+\left|u^{0}\right|^{2}+\left|u^{1}\right|^{2}+\beta \tau_{2}\left\|u^{1}\right\|^{2}+\frac{1}{(1-\varepsilon) \mu} \sum_{j=2}^{n} \frac{\tau_{j}}{1+r_{j}}\left\|f^{j}\right\|_{*}^{2}\right),
\end{gathered}
$$

which makes the application of Corollary 1 in Apendix A and thus sufficiently small $\tau_{\max }$ necessary. It follows (3.13) with the additional term $c \Lambda_{n} C_{1} t_{n}$ on the right-hand side and, instead of (3.14), with

$$
\Lambda_{n} \leq \frac{1}{1-c C_{1} \tau_{\max }} \exp \left(c \frac{\Gamma_{n}+C_{1} t_{n}+\gamma^{2} t_{n} / \mu}{1-c C_{1} \tau_{\max }}\right) .
$$

Suppose now that $g$ satisfies $\widetilde{(\mathrm{H} 1)}$. We then have for arbitrary $\varepsilon>0$

$2\left|\left\langle g\left(u^{n}\right), u_{\hat{\delta}}^{n}\right\rangle\right| \leq 2\left|g\left(u^{n}\right)\right|\left|u_{\hat{\delta}}^{n}\right| \leq 2 \widetilde{L}_{1}\left(1+\left\|u^{n}\right\|\right)\left|u_{\hat{\delta}}^{n}\right| \leq \varepsilon a\left(u^{n}, u^{n}\right)+1+\widetilde{C}_{1}\left|u_{\hat{\delta}}^{n}\right|^{2}$, where $\widetilde{C}_{1}=\widetilde{L}_{1}^{2}(1+4 /(\varepsilon \mu))$, and we do not need to change (3.8). Since

$$
\left|u_{\hat{\delta}}^{n}\right|^{2} \leq 2 \max \left(1, \hat{\delta}^{2}\right)\left(\left|u^{n}\right|^{2}+\left|u^{n}-u^{n-1}\right|^{2}\right) \leq c\left(\left|u^{n}\right|^{2}+\left|u^{n}-u^{n-1}\right|^{2}\right)
$$

for $1<R<\bar{R}$, we may take

$$
\begin{gathered}
K_{n}=c\left(\sum_{j=2}^{n-2}\left[r_{j+2}-r_{j}\right]_{-}\left(\left|u^{j}\right|^{2}+\left|u^{j}-u^{j-1}\right|^{2}\right)\right. \\
+\sum_{j=2}^{n-1}\left(\widetilde{C}_{1} \tau_{j}+\frac{\gamma^{2} \tau_{j+1}}{\mu}\right)\left(\left|u^{j}\right|^{2}+\left|u^{j}-u^{j-1}\right|^{2}\right)+\widetilde{C}_{1} \tau_{\max }\left(\left|u^{n}\right|^{2}+\left|u^{n}-u^{n-1}\right|^{2}\right) \\
\left.\quad+t_{n}+\left|u^{0}\right|^{2}+\left|u^{1}\right|^{2}+\beta \tau_{2}\left\|u^{1}\right\|^{2}+\frac{1}{\mu} \sum_{j=2}^{n} \frac{\tau_{j}}{1+r_{j}}\left\|f^{j}\right\|_{*}^{2}\right),
\end{gathered}
$$

and in virtue of (3.12), we can apply Corollary 1 in Appendix A if $\tau_{\max }$ is sufficiently small. We then find (3.13) with the additional term $c \Lambda_{n} t_{n}$ on the right-hand side and with $\Lambda_{n}$ as above changing $C_{1}$ to $\widetilde{C}_{1}$.

The dependence of the stability constant on problem parameters can be seen from the proof. 
Theorem 6. Let $f^{n}=\mathrm{R}_{2}^{n} f,\left\{u^{n}\right\} \in l^{\infty}(0, T ; H), u \in \mathcal{C}([0, T] ; H), f^{\prime}-u^{\prime \prime}$, and $f^{\prime \prime}-u^{\prime \prime \prime} \in L^{2}\left(0, T ; V^{*}\right)$. Assume (H2) (or, alternatively, ( $(\mathrm{H} 2)$ ). If $R<\bar{R}$ and $\tau_{\max }$ is sufficiently small, then the error $e^{n}$ to Problem $\left(P_{\tau}\right)$ satisfies (3.15).

Proof. We again commence with the error equation that reads now as

$$
\left(\mathrm{D}_{2} e^{n}, v\right)+a\left(e^{n}, v\right)+\left\langle g\left(u\left(t_{n}\right)\right)-g\left(u^{n}\right), v\right\rangle=\left\langle\rho^{n}, v\right\rangle \quad \forall v \in V .
$$

The consistency error $\rho^{n}$ to the associated linear problem is given by (3.16) or (3.17). Since $\left\{u^{n}\right\} \in l^{\infty}(0, T ; H)$ and $u \in \mathcal{C}([0, T] ; H)$, there is some $M>$ 0 , depending on problem data, such that $u\left(t_{n}\right), u^{n} \in \mathcal{B}_{M}(n=2,3, \ldots, N)$. Because of (H2), we have for arbitrary $\varepsilon \in(0,1)$,

$$
\begin{aligned}
2\left|\left\langle g\left(u\left(t_{n}\right)\right)-g\left(u^{n}\right), e_{\hat{\delta}}^{n}\right\rangle\right| & \leq 2 L_{2}(M)\left|e^{n}\right|^{s_{2}}\left\|e^{n}\right\|^{1-s_{2}}\left\|e_{\hat{\delta}}^{n}\right\| \\
& \leq C_{2}\left|e^{n}\right|^{2}+\varepsilon a\left(e^{n}, e^{n}\right)+\frac{\varepsilon}{2(1+\hat{\delta})} a\left(e_{\hat{\delta}}^{n}, e_{\hat{\delta}}^{n}\right),
\end{aligned}
$$

with some $C_{2}>0$ depending on $s_{2}, L_{2}(M)$, and $\varepsilon \mu$. Alternatively, we have with $\widetilde{(\mathrm{H} 2)}$ and some $\widetilde{C}_{2}>0$ depending on $\widetilde{L}_{2}(M)$ and $\varepsilon \mu$ that

$$
2\left|\left\langle g\left(u\left(t_{n}\right)\right)-g\left(u^{n}\right), e_{\hat{\delta}}^{n}\right\rangle\right| \leq 2 \widetilde{L}_{2}(M)\left\|e^{n}\right\|\left|e_{\hat{\delta}}^{n}\right| \leq \varepsilon a\left(e^{n}, e^{n}\right)+\widetilde{C}_{2}\left|e_{\hat{\delta}}^{n}\right|^{2} .
$$

We now follow the arguments in the proof of Theorem 5 and Theorem 4.

The error constant is essentially of the same structure as the stability constant (changing the subscript 1 to 2). The assumptions $u \in \mathcal{C}([0, T] ; H)$ and $\left\{u^{n}\right\} \in$ $l^{\infty}(0, T ; H)$ follow from Theorem 1 and Theorem 5 respectively if in particular (H1) (or $\widetilde{(\mathrm{H} 1)}$ ) holds true. These assumptions are only needed to ensure the existence of some $M>0$ independent of $\left\{\tau_{n}\right\}$ such that $u^{n}, u\left(t_{n}\right) \in \mathcal{B}_{M}(n=$ $2, \ldots, N)$, which enables to apply $(\mathrm{H} 2)$ (or $\widetilde{(\mathrm{H} 2)})$. The regularity assumptions can be only fulfilled under additional conditions on $g$.

\section{Appendix A: A discrete Gronwall lemma}

Lemma 1. Let $a_{n}, b_{n}, c_{n}, \lambda_{n} \geq 0$ with $\left\{c_{n}\right\}$ being monotonically increasing. Then

$$
a_{n}+b_{n} \leq \sum_{j=2}^{n-1} \lambda_{j} a_{j}+c_{n}, \quad n=2,3, \ldots
$$

implies for $n=2,3, \ldots$

$$
a_{n}+b_{n} \leq c_{n} \prod_{j=2}^{n-1}\left(1+\lambda_{j}\right) \leq c_{n} \exp \left(\sum_{j=2}^{n-1} \lambda_{j}\right) .
$$


Proof. With

$$
\tilde{a}_{m}:=\omega_{m-1} \sum_{j=2}^{m-1} \lambda_{j} a_{j}, \quad \omega_{m-1}:=\prod_{j=2}^{m-1}\left(1+\lambda_{j}\right)^{-1},
$$

for $m=2,3, \ldots$, we have

$$
\tilde{a}_{m+1}-\tilde{a}_{m}=\lambda_{m} \omega_{m}\left(a_{m}-\sum_{j=2}^{m-1} \lambda_{j} a_{j}\right) \leq c_{m} \lambda_{m} \omega_{m} .
$$

Summation gives (because of $\tilde{a}_{2}=0$ )

$$
\tilde{a}_{n} \leq \sum_{m=2}^{n-1} c_{m} \lambda_{m} \omega_{m} \leq c_{n} \sum_{m=2}^{n-1} \lambda_{m} \omega_{m} .
$$

We thus have from (A.1)

$$
a_{n}+b_{n} \leq \tilde{a}_{n} \omega_{n-1}^{-1}+c_{n} \leq c_{n} \omega_{n-1}^{-1}\left(\sum_{m=2}^{n-1} \lambda_{m} \omega_{m}+\omega_{n-1}\right),
$$

and the assertion follows with the identity

$$
\sum_{m=2}^{n-1} \lambda_{m} \omega_{m}+\omega_{n-1}=1
$$

Corollary 1. Let, in addition to the assumptions of Lemma 1, $0 \leq \lambda<1$. Then

$$
a_{n}+b_{n} \leq \sum_{j=2}^{n-1} \lambda_{j} a_{j}+\lambda a_{n}+c_{n}, \quad n=2,3, \ldots
$$

implies for $n=2,3, \ldots$

$$
a_{n}+b_{n} \leq \frac{c_{n}}{1-\lambda} \prod_{j=2}^{n-1}\left(1+\frac{\lambda_{j}}{1-\lambda}\right) \leq \frac{c_{n}}{1-\lambda} \exp \left(\frac{1}{1-\lambda} \sum_{j=2}^{n-1} \lambda_{j}\right)
$$

Proof. It immediately follows from (A.2) that

$$
a_{n}+b_{n} \leq a_{n}+\frac{b_{n}}{1-\lambda} \leq \sum_{j=2}^{n-1} \frac{\lambda_{j}}{1-\lambda} a_{j}+\frac{c_{n}}{1-\lambda},
$$

and we may apply Lemma 1 with $\lambda_{n}:=\lambda_{n} /(1-\lambda)$ and $c_{n}:=c_{n} /(1-\lambda)$. 


\section{Appendix B: Solvability of the continuous and discrete problem}

Before we are going to prove the solvability of both Problem $(P)$ and $\left(P_{\tau}\right)$, we provide the reader with some auxiliary results. We start with a well-known consequence of the famous fixed-point theorem by Brouwer.

Lemma 2. Let $\boldsymbol{h}: \mathbb{R}^{m} \rightarrow \mathbb{R}^{m}$ be continuous on $\mathcal{K}_{M}:=\left\{\boldsymbol{x} \in \mathbb{R}^{m}:\|\boldsymbol{x}\| \leq M\right\}$ for some $M>0$, where $\|\cdot\|$ denotes an arbitrary norm on $\mathbb{R}^{m}$. If $\boldsymbol{h}(\boldsymbol{x}) \cdot \boldsymbol{x} \geq 0$ for all $\boldsymbol{x} \in \mathbb{R}^{m}$ with $\|\boldsymbol{x}\|=M$, then there is some $\hat{\boldsymbol{x}} \in \mathcal{K}_{M}$ such that $\boldsymbol{h}(\hat{\boldsymbol{x}})=0$.

For a proof, see for instance Zeidler [21, Prop. 2.8].

Proposition 1. For $\tau>0$, let $B_{\tau}: V \rightarrow V^{*}$ be defined as

$$
B_{\tau}(v):=\frac{1}{\tau} v+A v+g(v), \quad v \in V .
$$

Assume (H1), (H2) (or, alternatively, $\widetilde{(\mathrm{H} 1)},(\widetilde{\mathrm{H} 2}))$, and $(\mathrm{H} 3)$. If $\tau$ is sufficiently small then $B_{\tau}$ is bijective.

Proof. We firstly observe that $B_{\tau}$ indeed maps $V$ into $V^{*}$ due to the properties of $A$ and $g$. Let $b \in V^{*}$ be arbitrary. We show by construction that $B_{\tau}(u)=b$ admits a unique solution $u \in V$. The existence of a solution will be proved by means of a Galerkin approximation: Since $V$ is separable, there is a Galerkin basis $\left\{\phi_{j}\right\} \subset V$ such that $\overline{\bigcup_{m \in \mathbb{N}} V_{m}}=V$ and $\lim _{m \rightarrow \infty} \operatorname{dist}\left(v, V_{m}\right)=0$ for all $v \in V$ with $V_{m}:=\operatorname{span}\left\{\phi_{1}, \ldots, \phi_{m}\right\}$. For given $m \in \mathbb{N}$, we consider the continuous and bijective mapping

$$
\boldsymbol{v}_{m}:=\left(v_{m, 1}, \ldots, v_{m, m}\right) \in \mathbb{R}^{m} \leftrightarrow v_{m}:=\sum_{j=1}^{m} v_{m, j} \phi_{j} \in V_{m}
$$

and introduce on $\mathbb{R}^{m}$ the norm $\left\|\boldsymbol{v}_{m}\right\|:=\left\|v_{m}\right\|$. The corresponding system of Galerkin equations

$$
\left\langle B_{\tau}\left(u_{m}\right), \phi_{j}\right\rangle=\left\langle b, \phi_{j}\right\rangle, \quad j=1, \ldots, m,
$$

is solvable if and only if there is a zero $\boldsymbol{u}_{m} \in \mathbb{R}^{m}$ of the function $\boldsymbol{h}: \mathbb{R}^{m} \rightarrow \mathbb{R}^{m}$ with $\boldsymbol{h}=\left(h_{1}, \ldots h_{m}\right)$ and

$$
h_{j}\left(\boldsymbol{v}_{m}\right):=\left\langle B_{\tau}\left(v_{m}\right)-b, \phi_{j}\right\rangle, \quad j=1, \ldots, m .
$$

Because of (H2) (or $\widetilde{(\mathrm{H} 2)}$ ) and (2.1), the function $\boldsymbol{h}$ is continuous on each $\mathcal{K}_{M}$ : In virtue of $V \hookrightarrow H$, it follows from $\boldsymbol{v}_{m} \in \mathcal{K}_{M}$ that $v_{m} \in \mathcal{B}_{\alpha M}$, and (H2) (or $\widetilde{(\mathrm{H} 2)})$ is applicable. We have furthermore

$$
\boldsymbol{h}\left(\boldsymbol{v}_{m}\right) \cdot \boldsymbol{v}_{m}=\left\langle B_{\tau}\left(v_{m}\right)-b, v_{m}\right\rangle=\frac{1}{\tau}\left|v_{m}\right|^{2}+a\left(v_{m}, v_{m}\right)+\left\langle g\left(v_{m}\right), v_{m}\right\rangle-\left\langle b, v_{m}\right\rangle
$$




$$
\geq \frac{1}{\tau}\left|v_{m}\right|^{2}+\mu\left\|v_{m}\right\|^{2}+\left\langle g\left(v_{m}\right), v_{m}\right\rangle-\|b\|_{*}\left\|v_{m}\right\| .
$$

With (H1) and Young's inequality, we find

$$
\begin{aligned}
& \left|\left\langle g\left(v_{m}\right), v_{m}\right\rangle\right| \leq\left\|g\left(v_{m}\right)\right\|_{*}\left\|v_{m}\right\| \\
& \leq L_{1}\left(1+\left|v_{m}\right|\right)^{s_{1}}\left\|v_{m}\right\|^{2-s_{1}} \leq C\left(1+\left|v_{m}\right|^{2}\right)+\frac{\mu}{2}\left\|v_{m}\right\|^{2},
\end{aligned}
$$

where $C$ depends on $L_{1}, s_{1}$, and $\mu$, and thus

$$
\boldsymbol{h}\left(\boldsymbol{v}_{m}\right) \cdot \boldsymbol{v}_{m} \geq\left(\frac{1}{\tau}-C\right)\left|v_{m}\right|^{2}+\frac{\mu}{2}\left\|v_{m}\right\|^{2}-\|b\|_{*}\left\|v_{m}\right\|-C .
$$

Taking $\tau \leq 1 / C$ and $M \geq\left(\|b\|_{*}+\sqrt{\|b\|_{*}^{2}+2 \mu C}\right) / \mu$, we have $\boldsymbol{h}\left(\boldsymbol{v}_{m}\right) \cdot \boldsymbol{v}_{m} \geq 0$ for all $\boldsymbol{v}_{m} \in \mathbb{R}^{m}$ with $\left\|\boldsymbol{v}_{m}\right\|=M$. With $\widetilde{(\mathrm{H} 1)}$, we find alternatively

$$
\begin{aligned}
& \left|\left\langle g\left(v_{m}\right), v_{m}\right\rangle\right| \leq\left|g\left(v_{m}\right)\right|\left|v_{m}\right| \\
& \quad \leq \widetilde{L}_{1}\left(1+\left\|v_{m}\right\|\right)\left|v_{m}\right| \leq C\left|v_{m}\right|^{2}+\frac{\mu}{2}\left\|v_{m}\right\|^{2}+\frac{\mu}{2},
\end{aligned}
$$

where $C$ depends on $\widetilde{L}_{1}$ and $\mu$. Taking $M \geq\left(\|b\|_{*}+\sqrt{\|b\|_{*}^{2}+\mu^{2}}\right) / \mu$ and $\tau \leq 1 / C$, we have again $\boldsymbol{h}\left(\boldsymbol{v}_{m}\right) \cdot \boldsymbol{v}_{m} \geq 0$ for all $\boldsymbol{v}_{m} \in \mathbb{R}^{m}$ with $\left\|\boldsymbol{v}_{m}\right\|=M$.

Lemma 2 now ensures the solvability of the system of Galerkin equations. Moreover, the sequence of Galerkin solutions $\left\{u_{m}\right\}$ is bounded in $V$ since $\left\|u_{m}\right\|=$ $\left\|\boldsymbol{u}_{m}\right\| \leq M$ with $M$ being independent of $m$. Since $V$ is a reflexive Banach space, there is a subsequence $\left\{u_{m^{\prime}}\right\}$ and some $u \in V$ such that $u_{m^{\prime}} \rightarrow u$ in $V$. We have to prove that $u$ solves $B_{\tau}(u)=b$. From the weak convergence, it follows that

$$
\left(\frac{1}{\tau} u_{m^{\prime}}, v\right) \rightarrow\left(\frac{1}{\tau} u, v\right), \quad\left\langle A u_{m^{\prime}}, v\right\rangle \rightarrow\langle A u, v\rangle
$$

for all $v \in V$. Because of (H3), also

$$
\left\langle g\left(u_{m^{\prime}}\right), v\right\rangle \rightarrow\langle g(u), v\rangle
$$

holds true for all $v \in V$. The assertion follows with the usual density argument.

It remains to prove the uniqueness: Let $u_{1}, u_{2} \in V$ be two solutions. We find

$$
\begin{aligned}
0 & =\left\langle B_{\tau}\left(u_{1}\right)-B_{\tau}\left(u_{2}\right), u_{1}-u_{2}\right\rangle \\
& \geq \frac{1}{\tau}\left|u_{1}-u_{2}\right|^{2}+\mu\left\|u_{1}-u_{2}\right\|^{2}+\left\langle g\left(u_{1}\right)-g\left(u_{2}\right), u_{1}-u_{2}\right\rangle .
\end{aligned}
$$

Taking $M:=\alpha \max \left(\left\|u_{1}\right\|,\left\|u_{2}\right\|\right)$, we have with (H2) and Young's inequality

$$
\begin{aligned}
\left|\left\langle g\left(u_{1}\right)-g\left(u_{2}\right), u_{1}-u_{2}\right\rangle\right| & \leq\left\|g\left(u_{1}\right)-g\left(u_{2}\right)\right\| *\left\|u_{1}-u_{2}\right\| \\
& \leq L_{2}\left|u_{1}-u_{2}\right|^{s_{2}}\left\|u_{1}-u_{2}\right\|^{2-s_{2}} \\
& \leq C\left|u_{1}-u_{2}\right|^{2}+\frac{\mu}{2}\left\|u_{1}-u_{2}\right\|^{2},
\end{aligned}
$$


where $C$ depends on $L_{2}=L_{2}(M), s_{2}$, and $\mu$. With $\widetilde{(\mathrm{H} 2)}$, we obtain analogously

$$
\begin{aligned}
\left|\left\langle g\left(u_{1}\right)-g\left(u_{2}\right), u_{1}-u_{2}\right\rangle\right| & \leq\left|g\left(u_{1}\right)-g\left(u_{2}\right)\right|\left|u_{1}-u_{2}\right| \\
& \leq \widetilde{L}_{2}\left|u_{1}-u_{2}\right|\left\|u_{1}-u_{2}\right\| \\
& \leq C\left|u_{1}-u_{2}\right|^{2}+\frac{\mu}{2}\left\|u_{1}-u_{2}\right\|^{2},
\end{aligned}
$$

where $C$ depends on $\widetilde{L}_{2}=\widetilde{L}_{2}(M)$ and $\mu$. It follows $u_{1}=u_{2}$ in $V$ for sufficiently small $\tau \leq 1 / C$.

Remark 3. As one infers from (B.2), Proposition 1 remains true, and indeed without a time step restriction, if instead of $(\mathrm{H} 1)$ or $\widetilde{(\mathrm{H} 1)}$ the function $g$ is positive on $V$ such that for all $v \in V$

$$
\langle g(v), v\rangle \geq 0 .
$$

We are now prepared to present the postponed proofs of Theorem 1 and 2 .

Proof of Theorem 1. We firstly observe that $A$ maps the space $L^{2}(0, T ; V)$ into its dual $L^{2}\left(0, T ; V^{*}\right)$. Because of $(\mathrm{H} 1)$ or $\widetilde{(\mathrm{H} 1)}$, also $g$ maps $L^{2}(0, T ; V)$ into $L^{2}\left(0, T ; V^{*}\right)$. Let $u \in L^{2}(0, T ; V)$ be a solution to Problem $(\mathrm{P})$. It then follows $u^{\prime}=f-A u-g(u) \in L^{2}\left(0, T ; V^{*}\right)$ and thus $u \in \mathcal{C}([0, T] ; H)$. In the following, we construct a solution and prove afterwards its uniqueness.

For given $N \in \mathbb{N}$, let $\tau=T / N$ and $t_{n}=n \tau(n=0, \ldots, N)$. We compute approximations $\left\{u^{n}\right\}$ by means of the implicit Euler method with constant step size, starting with $u^{0}=u_{0} \in H$ :

$$
\frac{u^{n}-u^{n-1}}{\tau}+A u^{n}+g\left(u^{n}\right)=f^{n}:=\frac{1}{\tau} \int_{t_{n-1}}^{t_{n}} f(t) d t, \quad n=1, \ldots, N .
$$

For sufficiently small $\tau>0$, Proposition 1 implies existence and uniqueness of a solution $\left\{u^{n}\right\}$ to (B.5). Testing by $u^{n}$, we find because of

$$
\left(u^{n}-u^{n-1}, u^{n}\right)=\frac{1}{2}\left(\left|u^{n}\right|^{2}-\left|u^{n-1}\right|^{2}+\left|u^{n}-u^{n-1}\right|^{2}\right)
$$

with (2.2) that

$$
\left|u^{n}\right|^{2}-\left|u^{n-1}\right|^{2}+\left|u^{n}-u^{n-1}\right|^{2}+2 \mu \tau\left\|u^{n}\right\|^{2} \leq 2 \tau\left\|f^{n}\right\|_{*}\left\|u^{n}\right\|-2 \tau\left\langle g\left(u^{n}\right), u^{n}\right\rangle .
$$

Estimates similar to (B.3) and (B.4) together with Young's inequality yield after summation

$$
\begin{aligned}
& \left|u^{n}\right|^{2}+\sum_{j=1}^{n}\left|u^{j}-u^{j-1}\right|^{2}+\mu \tau \sum_{j=1}^{n}\left\|u^{j}\right\|^{2} \\
& \leq\left|u^{0}\right|^{2}+\frac{2 \tau}{\mu} \sum_{j=1}^{n}\left\|f^{j}\right\|_{*}^{2}+C T+C \tau \sum_{j=1}^{n}\left|u^{j}\right|^{2} .
\end{aligned}
$$


With

$$
\tau \sum_{j=1}^{N}\left\|f^{j}\right\|_{*}^{2} \leq c \int_{0}^{T}\|f(t)\|_{*}^{2} d t
$$

Corollary 1 in Appendix A leads for sufficiently small $\tau$ to

$$
\left|u^{n}\right|^{2}+\sum_{j=1}^{n}\left|u^{j}-u^{j-1}\right|^{2}+\tau \sum_{j=1}^{n}\left\|u^{j}\right\|^{2} \leq C\left(\left|u_{0}\right|^{2}+\int_{0}^{T}\|f(t)\|_{*}^{2} d t+T\right) .
$$

Therefore, we have that $\left\{u^{n}\right\}$ is stable in $l^{2}(0, T ; V)$ and $l^{\infty}(0, T ; H)$. In addition, we obtain from (B.5) with (H1) or $\widetilde{(\mathrm{H} 1)}$ and with $(2.1)$

$$
\frac{1}{\tau}\left\|u^{n}-u^{n-1}\right\|_{*} \leq\left\|f^{n}\right\|_{*}+\beta\left\|u^{n}\right\|+\left\|g\left(u^{n}\right)\right\|_{*} \leq\left\|f^{n}\right\|_{*}+C\left\|u^{n}\right\|+C .
$$

Because of (B.6) and (B.7), it follows after summation

$$
\frac{1}{\tau} \sum_{j=1}^{N}\left\|u^{j}-u^{j-1}\right\|_{*}^{2} \leq C\left(\left|u_{0}\right|^{2}+\int_{0}^{T}\|f(t)\|_{*}^{2} d t+T\right),
$$

which shows that $\left\{\left(u^{n}-u^{n-1}\right) / \tau\right\}$ is in $l^{2}\left(0, T ; V^{*}\right)$. We now define for $t \in$ $\left(t_{n-1}, t_{n}\right](n=1, \ldots, N)$

$$
U_{\tau}(t):=u^{n}, \quad V_{\tau}(t):=\frac{u^{n}-u^{n-1}}{\tau}\left(t-t_{n-1}\right)+u^{n-1}
$$

with $U_{\tau}(0):=u^{1}, V_{\tau}(0)=u_{0}$. Let $\{\tau\}$ be a null sequence. Due to the stability of the discrete solution to (B.5), $\left\{U_{\tau}\right\}$ and $\left\{V_{\tau}\right\}$ are bounded in $L^{2}(0, T ; V)$ and $L^{\infty}(0, T ; H)$. However for the boundedness of $\left\{V_{\tau}\right\}$ in $L^{2}(0, T ; V)$, we need that $u_{0} \in V$. We can now extract a subsequence $\left\{\tau^{\prime}\right\}$ such that $\left\{U_{\tau^{\prime}}\right\}$ and $\left\{V_{\tau^{\prime}}\right\}$ are weakly convergent in $L^{2}(0, T ; V)$ and weakly* convergent in $L^{\infty}(0, T ; H)$. Moreover, the sequence of derivatives $\left\{V_{\tau}^{\prime}\right\}$ is bounded in $L^{2}\left(0, T ; V^{*}\right)$ because of (B.8). We, therefore, have due to the theorem by Lions and Aubin (see for instance Lions [12, Thm. 5.1 in Ch. 1.5.2]) the strong convergence of $\left\{V_{\tau^{\prime}}\right\}$ in $L^{2}(0, T ; H)$ and thus in any $L^{p}(0, T ; H)$ with $p \in[1, \infty)$. Since

$$
\int_{0}^{T}\left|U_{\tau}(t)-V_{\tau}(t)\right|^{2} d t=\frac{\tau}{3} \sum_{j=1}^{N}\left|u^{j}-u^{j-1}\right|^{2},
$$

we then have from (B.7) that also $\left\{U_{\tau^{\prime}}\right\}$ converges strongly in $L^{2}(0, T ; H)$ towards the same limit. We denote the limit by $u$ and have, finally, to prove that $u$ is a solution to the original problem. For this, we rewrite the scheme (B.5) as

$$
V_{\tau}^{\prime}(t)+A U_{\tau}(t)+g\left(U_{\tau}(t)\right)=F_{\tau}(t)
$$

for almost all $t \in(0, T)$ with $F_{\tau}(t)=f^{n}$ for $t \in\left(t_{n-1}, t_{n}\right](n=1, \ldots, N)$. For simplicity, we write $\tau$ instead of $\tau^{\prime}$. Testing by $v \in V$, multiplying by a test 
function $\phi=\phi(t) \in \mathcal{C}^{1}([0, T])$ with $\phi(T)=0$, and integration leads to

$$
\begin{aligned}
& -\int_{0}^{T}\left(V_{\tau}(t), v\right) \phi^{\prime}(t) d t+\int_{0}^{T} a\left(U_{\tau}(t), v\right) \phi(t) d t+\int_{0}^{T}\left\langle g\left(U_{\tau}(t)\right), v\right\rangle \phi(t) d t \\
& =\int_{0}^{T}\left\langle F_{\tau}(t), v\right\rangle \phi(t) d t+\left(u_{0}, v\right) \phi(0) .
\end{aligned}
$$

Due to the strong convergence of $\left\{V_{\tau}\right\}$ in $L^{2}(0, T ; H)$ and the weak convergence of $\left\{U_{\tau}\right\}$ in $L^{2}(0, T ; V)$, we have that

$$
\begin{aligned}
\int_{0}^{T}\left(V_{\tau}(t), v\right) \phi^{\prime}(t) d t & \rightarrow \int_{0}^{T}(u(t), v) \phi^{\prime}(t) d t, \\
\int_{0}^{T} a\left(U_{\tau}(t), v\right) \phi(t) d t & \rightarrow \int_{0}^{T} a(u(t), v) \phi(t) d t .
\end{aligned}
$$

Since $U_{\tau}, u \in L^{\infty}(0, T ; H)$, there is some $M>0$ such that $U_{\tau}(t), u(t) \in \mathcal{B}_{M}$ for almost all $t \in(0, T)$. With (H2) and Hölder's inequality, it then follows because of the strong convergence in $L^{2}(0, T ; H)$ and the boundedness in $L^{2}(0, T ; V)$ that

$$
\begin{aligned}
& \int_{0}^{T}\left|\left\langle g\left(U_{\tau}(t)\right)-g(u(t)), v\right\rangle \phi(t)\right| d t \\
& \leq L_{2} \max _{t \in[0, T]}|\phi(t)|\|v\| \int_{0}^{T}\left|U_{\tau}(t)-u(t)\right|^{s_{2}}\left\|U_{\tau}(t)-u(t)\right\|^{1-s_{2}} d t \\
& \leq L_{2} \max _{t \in[0, T]}|\phi(t)|\|v\| \int_{0}^{T}\left|U_{\tau}(t)-u(t)\right| d t \int_{0}^{T}\left\|U_{\tau}(t)-u(t)\right\| d t \rightarrow 0 .
\end{aligned}
$$

Unfortunately, $\widetilde{(\mathrm{H} 2)}$ is not enough to conclude the desired convergence. However, we have alternatively $\widetilde{(\mathrm{H} 3)}$ at hand. With standard arguments, we may prove $F_{\tau} \rightarrow f$ in $L^{2}\left(0, T ; V^{*}\right)$, and so it follows that $u$ is a weak solution to (2.4).

Regarding the restriction to initial conditions $u_{0} \in V$, we note that if only $u_{0} \in H$, then we only have the boundedness of $\left\{V_{\tau}\right\}$ in $L^{2}(\varepsilon, T ; V)$ for any $\varepsilon \geq \tau$. It follows strong convergence of $\left\{V_{\tau}\right\}$ in $L^{2}(\varepsilon, T ; H)$. Since $\left\{V_{\tau}\right\}$ remains bounded in $L^{\infty}(0, T ; H)$, it also follows strong convergence in $L^{2}(0, T ; H)$.

It remains to prove the uniqueness. Let $u_{1}, u_{2}$ be two solutions. Then $w:=$ $u_{1}-u_{2} \in L^{2}(0, T ; V) \cap \mathcal{C}([0, T] ; H)$ with $w^{\prime} \in L^{2}\left(0, T ; V^{*}\right)$, and

$$
\left\langle w^{\prime}(t), v\right\rangle+a(w(t), v)=-\left\langle g\left(u_{1}(t)\right)-g\left(u_{2}(t)\right), v\right\rangle
$$

holds for all $v \in V$ and almost everywhere in $(0, T)$. Taking $v=w(t)$, we find with (H2) and Young's inequality

$$
\begin{gathered}
\frac{1}{2} \frac{d}{d t}|w(t)|^{2}+\mu\|w(t)\|^{2} \leq\left\|g\left(u_{1}(t)\right)-g\left(u_{2}(t)\right)\right\|_{*}\|w(t)\| \\
\leq L_{2}|w(t)|^{s_{2}}\|w(t)\|^{2-s_{2}} \leq \frac{C}{2}|w(t)|^{2}+\frac{\mu}{2}\|w(t)\|^{2},
\end{gathered}
$$


where $C$ depends on $L_{2}=L_{2}(M), s_{2}$, and $\mu$ with

$$
M:=\max \left(\max _{t \in[0, T]}\left|u_{1}(t)\right|, \max _{t \in[0, T]}\left|u_{2}(t)\right|\right) .
$$

Alternatively, we obtain from $\widetilde{(\mathrm{H} 2)}$

$$
\begin{gathered}
\frac{1}{2} \frac{d}{d t}|w(t)|^{2}+\mu\|w(t)\|^{2} \leq\left|g\left(u_{1}(t)\right)-g\left(u_{2}(t)\right)\right||w(t)| \\
\leq \widetilde{L}_{2}|w(t)|\|w(t)\| \leq \frac{C}{2}|w(t)|^{2}+\frac{\mu}{2}\|w(t)\|^{2},
\end{gathered}
$$

where $C$ depends on $\widetilde{L}_{2}=\widetilde{L}_{2}(M)$ and $\mu$. In both cases, we come up with

$$
\frac{d}{d t}\left(\mathrm{e}^{-C t}|w(t)|^{2}\right)+\mu \mathrm{e}^{-C t}\|w(t)\|^{2} \leq 0,
$$

which proves, after integration, the uniqueness since $w(0)=0$.

Proof of Theorem 2. Let $u^{n-2}, u^{n-1} \in V^{*}$ and $f^{n} \in V^{*}$ be given. We rewrite the $n$-th step $(n=2, \ldots, N)$ of the BDF in the form $B_{\tau}\left(u^{n}\right)=b$, where $B_{\tau}$ is given by (B.1) with

$$
\tau:=\frac{\tau_{n}\left(1+r_{n}\right)}{1+2 r_{n}}, \quad b:=f^{n}+\frac{1}{\tau_{n}}\left(\left(1+r_{n}\right) u^{n-1}-\frac{r_{n}^{2}}{1+r_{n}} u^{n-2}\right) .
$$

From Proposition 1, we conclude existence and uniqueness of $u^{n} \in V \subset V^{*}$ if $\tau_{n}$ is sufficiently small. This proves the unique solvability of Problem $\left(P_{\tau}\right)$ for given $u^{0}, u^{1} \in H \subset V^{*},\left\{f^{n}\right\} \subset V^{*}$ if $\tau_{\max }$ is sufficiently small.

\section{REFERENCES}

1. J. Becker, A second order backward difference method with variable steps for a parabolic problem, BIT, 38 (1998) 4, pp. $644-662$.

2. M. Crouzeix and V. Thomée, On the discretization in time of semilinear parabolic equations with nonsmooth initial data, Math. Comp., 49 (1987) 180, pp. 359 - 377.

3. E. Emmrich, Stability and convergence of the two-step BDF for the incompressible NavierStokes problem, Int. J. Nonlinear Sci. Numer. Simul., 5 (2004) 3, pp. 199 - 209.

4. E. Emmrich, Error of the two-step BDF for the incompressible Navier-Stokes problem, M2AN, Math. Model. Numer. Anal., 38 (2004) 5, 757 - 764.

5. D. Estep and S. Larsson, The discontinuous Galerkin method for semilinear parabolic problems, M2AN, Math. Model. Numer. Anal., 27 (1993) 1, 35 - 54.

6. H. Fujita and T. Suzuki, Evolution Problems, in Handbook of Numerical Analysis, Vol. II: Finite Element Methods (Part 1), P. G. Ciarlet and J.-L. Lions, eds., Elsevier, Amsterdam, 1991, pp. $789-928$.

7. C. González, A. Ostermann, C. Palencia, and M. Thalhammer, Backward Euler discretization of fully nonlinear parabolic problems, Math. Comput., 71 (2002) 237, pp. 125 - 145. 
8. R. D. Grigorieff, Stability of multistep-methods on variable grids, Numer. Math., 42 (1983), pp. $359-377$.

9. R. D. Grigorieff, Time discretization of semigroups by the variable two-step BDF method, in Numerical treatment of differential equations, Sel. Papers NUMDIFF-5, Halle, 1989, B. G. Teubner, Leipzig, 1991, pp. $204-216$.

10. R. D. Grigorieff, On the variable grid two-step BDF method for parabolic equations, Preprint 426, Fachber. Mathematik, Techn. Univ. Berlin, 1995.

11. M.-N. Le Roux, Variable step size multistep methods for parabolic problems, SIAM J. Numer. Anal., 19 (1982) 4, pp. 725 - 741

12. J.-L. Lions, Quelques méthodes de résolution des problèmes aux limites non linéaires, Dunod Gauthier-Villars, Paris, 1969

13. C. Lubich and A. Ostermann, Linearly implicit time discretization of non-linear parabolic equations, IMA J. Numer. Anal., 15 (1995) 4, pp. 555 - 583.

14. C. Lubich and A. Ostermann, Runge-Kutta time discretization of reaction-diffusion and Navier-Stokes equations: nonsmooth-data error estimates and applications to long-time behaviour, Appl. Numer. Math., 22 (1996), pp. 279 - 292.

15. R. Temam, Behaviour at time $t=0$ of the solutions of semi-linear evolution equations, J. Diff. Eqs., 43 (1982), pp. $73-92$.

16. A. Ostermann and M. Thalhammer, Non-smooth data error estimates for linearly implicit Runge-Kutta methods, IMA J. Numer. Anal., 20 (2000) 20, pp. 167 - 184

17. C. Palencia and B. García-Archilla, Stability of linear multistep methods for sectorial operators in Banach spaces, Appl. Numer. Math., 12 (1993) 6, pp. 503 - 520.

18. M. Slodička, Smoothing effect and discretization in time to semilinear parabolic equations with nonsmooth data, Comment. Math. Univ. Carol., 32 (1991) 4, $703-713$.

19. M. Slodička, Semigroup formulation of Rothe's method: application to parabolic problems, Comment. Math. Univ. Carol., 33 (1992) 2, 245 - 260.

20. V. Thomée, Galerkin Finite Element Methods for Parabolic Problems, Springer, Berlin, 1997.

21. E. Zeidler, Nonlinear Functional Analysis and its Applications I, Springer, New York, 1992.

22. M. Zlámal, Finite element methods for nonlinear parabolic equations, RAIRO Analyse Numérique, 11 (1977) 1, pp. 93 - 107.

Etienne Emmrich received his Diploma degree in mathematics from the Magdeburg University in Germany and his Dr. rer. nat. from the Technical University Berlin. In his research, he is in particular interested in the numerical analysis of differential equations.

Technische Universität Berlin, Institut für Mathematik, Straße des 17. Juni 136, 10623 Berlin, Germany

e-mail: emmrich@math.tu-berlin.de 